\title{
Tumor neuroendócrino de apêndice: estudo de caso
}

\author{
Appendix neuroendocrine tumor: case study \\ Tumor neuroendocrino del apéndice: estudio de caso
}

Igor Vitoi Cangussu', Edna Delabio Ferraz², Luiz Felipe Costa Mello', Cristina Pimont de Oliveira1, Leatrice Schubert Guilhon de Castro', Thaís Bandeira de Oliveira Junqueira', Thais Rezende Reis ${ }^{3}$, Arthur Valdier Batista', Lucas Machado de Souza Vicente" ${ }^{4 *}$, Ana Luíza de Castro Carvalho ${ }^{5}$.

\begin{abstract}
RESUMO
Objetivo: Este artigo visa relatar um caso em que houve diagnóstico acidental de tumor neuroendócrino do apêndice no decorrer de investigação tomográfica de cálculo renal. Detalhamento do caso: Mulher, 62 anos, com diagnóstico acidental, em tomografia computadorizada, de tumoração em apêndice cecal sendo submetida a apendicectomia videolaparoscópica com diagnóstico anatomopatológico (AP) e imuno-histoquímico de tumor neuroendócrino de $2,5 \times 1,5 \mathrm{~cm}$, infiltrando gordura adjacente, com infiltração perineural, positivo para cromogranina e índice proliferativo pelo Ki $67<2 \%$. Devido ao achado do AP a paciente foi submetida à hemicolectomia direita. A paciente em questão permaneceu em acompanhamento por 2 anos, sendo realizado nesse período tomografia computadorizada seriada anualmente; em seu último controle no ambulatório foi orientada e liberada do acompanhamento, com cura do quadro. Considerações Finais: Os tumores neuroendócrinos do apêndice são neoplasias raras, geralmente com bom prognóstico. Seu diagnóstico e tratamento devem ser baseados no laudo histopatológico e imuno-histoquímico. Em casos selecionados, está indicada a hemicolectomia direita.
\end{abstract}

Palavras-chave: Carcinoma neuroendócrino, Neoplasias do apêndice, Apêndice.

\section{ABSTRACT}

Objective: This article aims to report a case in which there was an accidental diagnosis of neuroendocrine tumor of the appendix during tomographic investigation of renal calculus. Case Report: Woman, 62 years old, with accidental diagnosis, on computed tomography, of tumor in the cecal appendix undergoing videolaparoscopic appendectomy with anatomopathological diagnosis (AP) and immunohistochemistry of a $2.5 \times 1.5 \mathrm{~cm}$ neuroendocrine tumor, infiltrating fat adjacent, with perineural infiltration, positive for chromogranin and proliferative index by Ki67 <2\%. Due to the finding of the AP, she underwent a right hemicolectomy. The patient in question remained in follow-up for 2 years, with serial computerized tomography performed annually; in her last check-up at the clinic, she was instructed and released from follow-up, with a cure. Final Considerations: Neuroendocrine tumors of the appendix are rare neoplasms, usually with a good prognosis. Its diagnosis and treatment must be based on the histopathological and immunohistochemical report. In selected cases, right hemicolectomy is indicated.

Keywords: Carcinoma neuroendocrine, Appendiceal neoplasms, Appendix.

\section{RESUMEN}

Objetivo: Este artículo tiene como objetivo reportar un caso en el que hubo un diagnóstico accidental de tumor neuroendocrino de apéndice durante la exploración tomográfica de cálculo renal. Caso clínico: Mujer de 62 años, con diagnóstico accidental, en tomografía computarizada, de tumor en apéndice cecal siendo sometida a apendicectomía videolaparoscópica con diagnóstico anatomopatológico (DA) e inmunohistoquímica de tumor neuroendocrino de $2,5 \times 1,5 \mathrm{~cm}$, infiltrante de grasa adyacente, con infiltración perineural, positivo para cromogranina e índice de proliferación por Ki67 <2\%. Debido al hallazgo de la DA, se le realizó una hemicolectomía derecha. El paciente en cuestión permaneció en seguimiento durante 2 años, realizándose anualmente una

\footnotetext{
${ }^{1}$ Hospital e Maternidade Therezinha de Jesus (HMTJ), Juiz de Fora - MG. *E-mail: lucasvicente.med@gmail.com

${ }^{2}$ Universidade Federal Fluminense (UFF), Rio de Janeiro - RJ.

${ }^{3}$ Santa Casa de Misericórdia de Juiz de Fora, Juiz de Fora - MG.

${ }^{4}$ Faculdade de Ciências Médicas e da Saúde de Juiz de Fora (FCMS/JF), Juiz de Fora - MG.

${ }^{5}$ Universidade Federal de Juiz de Fora (UFJF), Juiz de Fora - MG.
} 
tomografía computarizada seriada; en su último control en la consulta externa, fue instruida y dada de alta del seguimiento, con cura. Consideraciones finales: Los tumores neuroendocrinos de apéndice son neoplasias raras, habitualmente con buen pronóstico. Su diagnóstico y tratamiento debe basarse en el informe histopatológico e inmunohistoquímico. En casos seleccionados, está indicada la hemicolectomía derecha.

Palabras Clave: Carcinoma neuroendocrino, Neoplasias del apéndice, Apéndice.

\section{INTRODUÇÃO}

Os tumores neuroendócrinos (TNE) são uma entidade do apêndice que compreendem tumores raros, porém representam as neoplasias mais comuns do apêndice (MODLIN IM e SANDOR A, 1997), com taxa de aproximadamente entre 80 e 88\% (MORIS D, et al., 2018; CORREA NETO IJF, et al., 2014).

O apêndice corresponde ao terceiro sítio mais comum de TNE do trato gastrointestinal (TGI) (17\%), atrás apenas do intestino delgado (45\%) e do reto (20\%) (GROZINSKY-GLASBERG S, et al., 2013). Esses tumores são mais comuns em mulheres (2-4:1) com idade entre 38 e 51 anos, variando entre os artigos estudados, sem predileção por raça (CORREA NETO IJF, et al., 2014).

O diagnóstico pré-operatório dos TNE do apêndice é raro, geralmente realizado de forma incidental, por achado em exame histopatológico de pacientes submetidos a apendicectomia por apendicite aguda ou outras cirurgias abdominais. A incidência é de aproximadamente 3 a 5 pacientes a cada 1.000 operados, podendo chegar a 1 caso em 100 pacientes em alguns estudos (PAPE UF, et al., 2016; CORREA NETO IJF, et al., 2014; SILVA RL, et al., 2010) e a 1\% das amostras de apendicectomias (CONNOR SJ, et al., 1998).

A maior parte dos casos são assintomáticos, e quando sintomáticos a apresentação clínica é geralmente inespecífica (BAYHAN Z, et al., 2020). Geralmente se localizam no terço distal do apêndice, onde é praticamente improvável o desenvolvimento de quadro obstrutivo, por isso não são necessariamente associados a clínica típica de apendicite aguda (SILVA RL, et al., 2010).

Anteriormente denominados carcinoides, os TNE do apêndice se originam de células progenitoras na submucosa e lâmina própria, e se localizam sobretudo (75\%) no ápice do órgão, devido à grande concentração de células neuroendócrinas subepiteliais na região distal (SHAW PA, 1991). Estes tumores, diferentemente dos TNE do intestino anterior e posterior, podem secretar hormônios, como gastrina, substância P, calcitonina e insulina, porém sem manifestação clínica (SPANOS CP e KAISER AM, 2016). A apresentação típica engloba nódulos de coloração amarelada e firmes, sendo a maioria dos tumores menores que $1 \mathrm{~cm}$, além de a análise histopatológico evidenciar conglomerados celulares uniformes submucosos e núcleos em padrão de cromatina endócrina de "sal e pimenta" (HATCH QM e GILBERT EW, 2018).

A maioria dos pacientes com diagnóstico de TNE do apêndice apresenta curso indolente e bom prognóstico sendo a apendicectomia o único tratamento necessário (PAPE UF, et al., 2016; CORREA NETO IJF, et al., 2014; GROZINSKY-GLASBERG S, et al., 2013), com taxas de sobrevida maiores que $90 \%$ em 5 anos (GAETKE-UDAGER K, et al., 2014).

Porém, em alguns casos com maior risco de doença metastática, a hemicolectomia direita está indicada. Dentre os fatores que auxiliam o estabelecimento do plano terapêutico são citados tamanho do tumor, localização, invasão do mesoapêndice, invasão vascular e presença de metástase a distância (CORREA NETO IJF, et al., 2014; WILLIAM Z, et al., 2013; PAPE UF, et al., 2012).O objetivo do presente artigo foi analisar, de forma concisa e clara, um estudo de caso, que é raro na literatura, sobre TNE do apêndice e seus principais aspectos.

\section{DETALHAMENTO DO CASO}

Paciente do sexo feminino, 62 anos de idade, em acompanhamento devido a quadro de litíase renal, realizou tomografia computadorizada de abdome e pelve para controle em agosto de 2015, que evidenciou tumoração em apêndice cecal. Após estadiamento que demonstrou a presença de doença apenas localizada, optou-se pela realização de tratamento cirúrgico. 
Realizada apendicectomia videolaparoscópica, em janeiro de 2016, com identificação de tumoração em segmento distal do apêndice, não sendo possível a realização de exame de congelação por falta de condições operacionais. A cirurgia ocorreu sem intercorrências e paciente apresentou boa evolução (Figura 1, Figura 2). O laudo histopatológico descreve um tumor neuroendócrino do apêndice cecal constituído por ninhos sólidos de células monótonas com ocasional formação de ácinos ou rosetas, apresentando baixo índice mitótico, e neoplasia com dimensões de $2,5 \mathrm{~cm} \times 1,5 \mathrm{~cm}$, infiltrando até a serosa.

A margem cirúrgica se encontrava livre de neoplasia. Constatada presença de infiltração perineural, porém não foi identificado invasão angiolinfática inequívoca. O Material enviado para estudo imuno-histoquímico que revelou tratar-se de um tumor neuroendócrino grau 1, com infiltração de toda a parede do órgão e comprometimento da gordura adjacente (infiltração da gordura medindo $0,45 \mathrm{~mm}$ ). Revelou positividade para cromogranina e índice proliferativo pelo Ki- $67<2 \%$, sendo a classificação patológica de pT2 pela classificação The American Joint Committee on Cancer (AJCC) e pT3 pela classificação European Neuroendocrine Tumor Society (ENETS) (Tabela 1) (PAPE UF, et al., 2016).

Em função dos achados acima, optou-se por complementar o tratamento com hemicolectomia direita videolaparoscópica devido ao tamanho do tumor. O procedimento foi realizado três meses após a primeira cirurgia, sem intercorrências. O paciente recebeu alta no terceiro dia de pós-operatório, em bom estado geral. Retornou ao hospital após três dias com quadro de constipação com boa resposta após medidas clínicas. $O$ laudo histopatológico evidenciou presença de processo inflamatório sem sinais de neoplasia na peça cirúrgica. No oitavo mês de seguimento, o paciente não apresentava sinais de recidiva.

A paciente em questão permaneceu em acompanhamento por 2 anos, sendo realizado nesse período tomografia computadorizada de abdome seriada anualmente; em seu último controle no ambulatório foi devidamente orientada e liberada do acompanhamento, com cura total do quadro.

Figura 1 - Tomografia computadorizada de abdome e pelve mostrando cálculo em pelve renal direita.

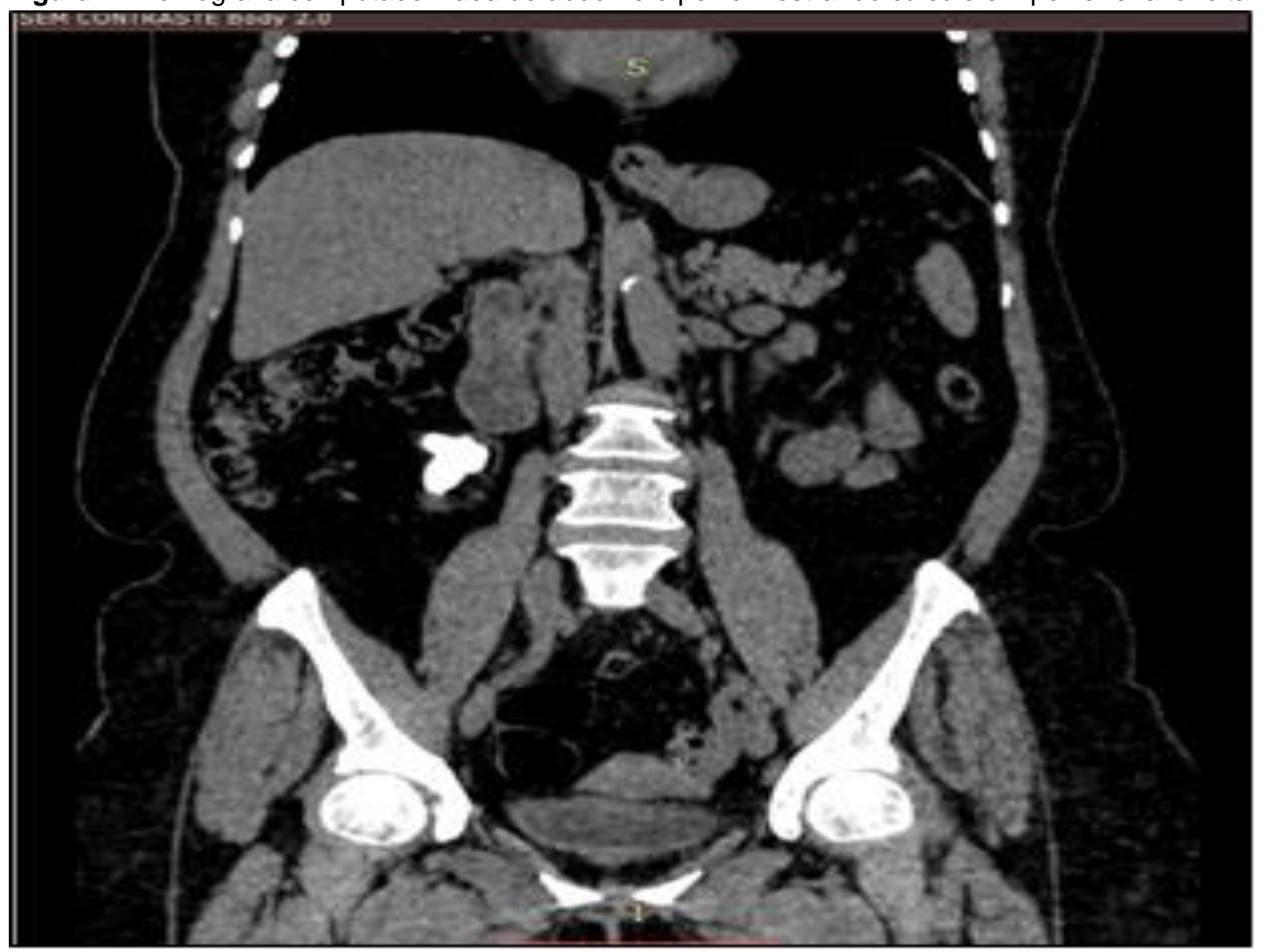

Fonte: CANGUSSU IV, et al., 2020. 
Figura 2 - Tomografia computadorizada de abdome e pelve apresentando tumoração em apêndice cecal (seta vermelho).

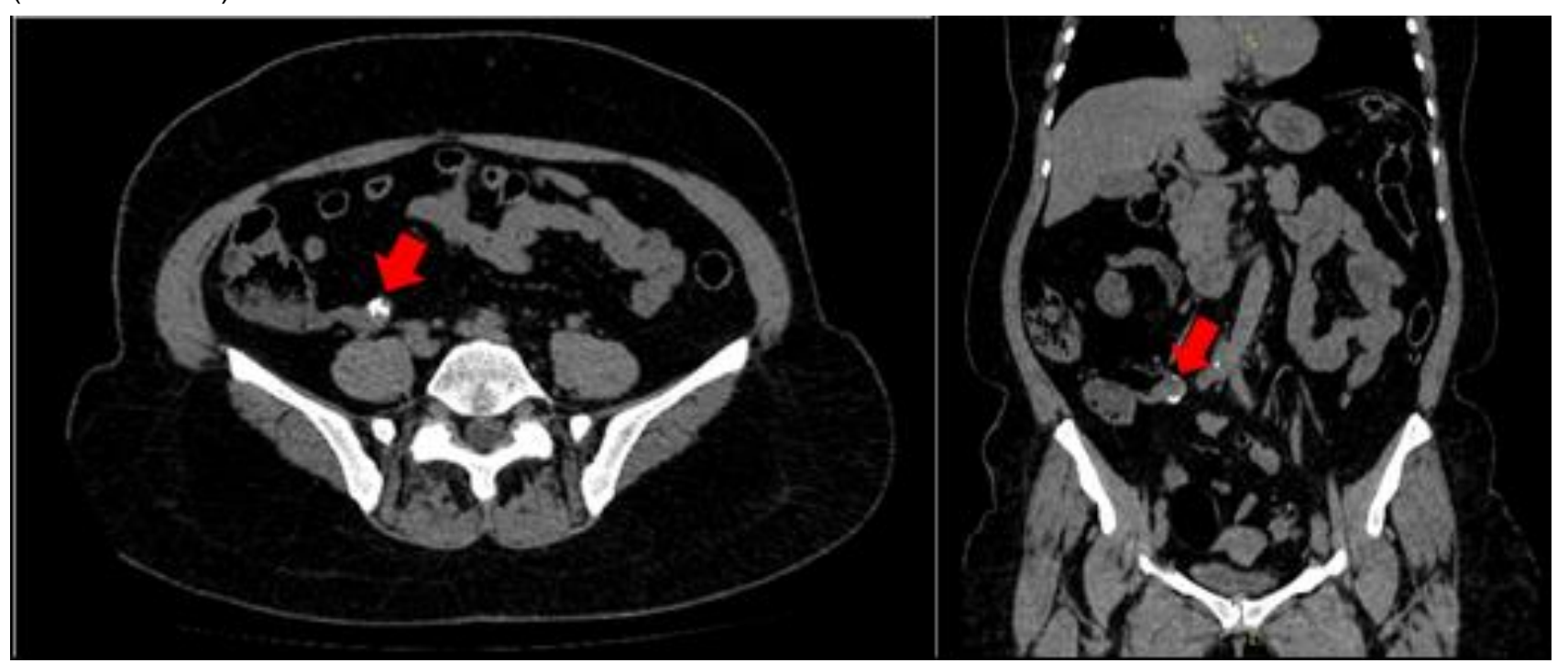

Fonte: CANGUSSU IV, et al., 2020.

\section{DISCUSSÃO}

As células neuroendócrinas estão distribuídas por todo o corpo e podem ser encontradas no TGI, pulmões, pâncreas, tireoide, entre outros. No TGI, os tumores ocorrem principalmente no intestino delgado (45\%), reto (20\%), apêndice (17\%), cólon (10,6\%) e estômago (7,2\%) (TOMIOKA K, et al., 2013). Os TNE do apêndice têm uma incidência na literatura de 0.15-0.6 em 100.000 por ano, com uma pequena predisposição ao sexo feminino e em pacientes com idade entre 38 e 51 anos (PAPE UF, et al., 2016). São mais frequentes em parte distal do apêndice (75\%) e menos frequentes nos terços médio (15\%) e proximal (10\%) (WILLIAM Z, et al., 2013).

Os TNE do apêndice são diagnosticados, na maioria das vezes, incidentalmente durante o perioperatório de apendicectomias ou na investigação ou tratamento de outras condições abdominais (ALEXANDRAKI KI, et al., 2016). Nas faixas etárias pediátricas e juvenis há maiores chances de manifestação desta patologia através de quadro característico de abdome agudo, em contrapartida, nos pacientes adultos são geralmente identificadas de forma incidental através de exames de imagens realizados para investigação de outra suspeita diagnóstica ou do exame anatomopatológico da peça cirúrgica da apendicectomia (MOERTEL CL, et al.,1990). Em pacientes submetidos a apendicectomia a taxa de incidência de TNE varia de 0,3 a 0,9\% (ALEXANDRAKI KI, et al., 2016)

O diagnóstico pré-operatório é considerado difícil devido à falta de sinais e/ou sintomas específicos que permitam diferir de um quadro de apendicite aguda (CORREA NETO IJF, et al., 2014; FERNANDES LC, et al., 2002; MORIS D, et al., 2018; SILVA RL, et al., 2010). Por isso, na maioria dos casos, é diagnosticado incidentalmente no exame histopatológico após o tratamento cirúrgico de suposta apendicite (MORIS D, et al., 2018), que evidencia células tumorais uniformes, dispostas em ninhos arredondados e sólidos, sendo a maioria tumores de células enterocromafins produtoras de serotonina (DESCHAMPS D e COUVELARD A, 2010).

Apresenta uma incidência variando de 3/1.0003,4 até 1/1002 em pacientes operados. A biópsia de congelação deve ser realizada em casos de apêndice atípico visando evitar uma segunda intervenção (TOMIOKA K, et al., 2013; LOPES JÚNIOR AG, et al., 2001). Além do exame histopatológico é necessário também a imuno-histoquímica tanto para confirmação diagnóstica, principalmente com o uso da cromogranina (marcador tumoral dos TNEs, quanto para avaliar prognóstico com a dosagem dos níveis de Ki-67 relacionado com a capacidade de proliferação tumoral (PAPE UF, et al., 2016; CORREA NETO IJF, et al., 2014; SAHAN EK, et al., 2015; SILVA RL, et al., 2010). 
Pode ser classificada como baixa (<2\%), de melhor prognóstico, moderada (2 - 20\%) e alta ( $>20 \%)$, de pior prognóstico (CORREA NETO IJF, et al., 2014; SAHAN EK, et al., 2015). A colonoscopia é indicada como exame complementar em casos confirmados, devido ao fato de até $18 \%$ dos pacientes apresentarem neoplasia no TGI concomitante (BOUDREAUX JP, et al., 2010). No caso apresentado, a paciente apresentava resultado indicativo baixa proliferação tumoral.

Mediante o diagnóstico histopatológico é preconizada a análise de parâmetros tumorais como preditor de prognóstico: tamanho, localização, invasão, infiltração no órgão e índice de proliferação (Ki-67). Essas medidas devem constar no relatório histopatológico, pois são utilizadas na estimativa do potencial de agressividade do tumor, da chance de recidiva loco-regional e da ocorrência de metástase a distância (MORIS D, et al., 2018).

As taxas de sobrevida dos TNE do apêndice são melhores que as encontradas em outros TNEs gastrointestinais (DASARI A, et al., 2017). Em geral, os TNE do apêndice costumam ter bom prognóstico, sendo geralmente operados em fases iniciais com uma sobrevida em 5 anos perto de $100 \%$ em pacientes com doença local, de 85 a 100\% em pacientes com doença locorregional, e pior prognóstico nos raros casos de metástase a distância com uma sobrevida em 5 anos de 12 a 28\% (PAPE UF, et al., 2016; AMR B, et al., 2015).

Em consonância, alguns estudos e a análise do banco de dados Surveillance, Epidemiology and End Results (SEER) encontraram as seguintes taxas de sobrevida durante 5 anos: $94 \%$ para lesões confinadas, 84,6\% para acometimento locorregional e 33,7\% para presença de metástase à distância (MODLIN IM e SANDOR A, 1997; SANDOR A e MODLIN IM, 1998).

A chave para o tratamento ideal é a correta avaliação do tumor, sendo as classificações mais utilizadas a classificação da ENETES e a do AJCC (Quadro 1) (PAPE UF, et al., 2016). Nas fases iniciais, os tratamentos preconizados são a apendicectomia ou a hemicolectomia direita, a depender da análise de demais fatores (YAO JC, et al., 2008). Em estágios mais avançados é indicada cirurgia oncológica ampliada e consideradas terapias complementares (MORIS D, et al., 2018).

Quadro 1 - Classificação TNM pela The American Joint Committee on Cancer (AJCC) e pela European Neuroendocrine Tumors Society (ENETS).

\begin{tabular}{|c|c|}
\hline ENETS & AJCC \\
\hline \multicolumn{2}{|c|}{ Tx: Tumor primário não pode ser avaliado } \\
\hline \multicolumn{2}{|c|}{ T0: Sem evidência de tumor } \\
\hline \multirow{2}{*}{$\mathrm{T} 1: \leq 1 \mathrm{~cm}$ invadindo até muscular própria } & T1a: $\leq 1 \mathrm{~cm}$ \\
\hline & T1b: $>1 \mathrm{~cm} \mathrm{e} \leq 2 \mathrm{~cm}$ \\
\hline $\begin{array}{c}\text { T2: } \leq 2 \mathrm{~cm} \text { invadindo até muscular própria e/ou } \\
\text { invasão mínima (até } 3 \mathrm{~mm} \text { ) de } \\
\text { subserosa/mesoapêndice }\end{array}$ & T2: > $2 \mathrm{~cm}$ e $\leq 4 \mathrm{~cm}$ ou extensão para ceco \\
\hline $\begin{array}{c}\text { T3: }>2 \text { cm e/ou invasão extensa }(>3 \mathrm{~mm}) \text { de } \\
\text { subserosa/mesoapêndice }\end{array}$ & T3: > 4 cm ou extensão para íleo \\
\hline T4: Invasão peritoneal ou outros órgãos & $\begin{array}{l}\text { T4: Tumor perfurado ou invasão de órgãos ou } \\
\text { estruturas adjacentes }\end{array}$ \\
\hline \multicolumn{2}{|c|}{ Nx: Linfonodos regionais não podem ser avaliados } \\
\hline \multicolumn{2}{|c|}{ N0: Sem metástase para linfonodos regionais } \\
\hline \multicolumn{2}{|c|}{ N1: Com metástase para linfonodos regionais } \\
\hline \multicolumn{2}{|c|}{ Mx: Não se pode avaliar metástases a distância } \\
\hline \multicolumn{2}{|c|}{ M0: Nenhuma metástase a distância } \\
\hline \multicolumn{2}{|c|}{ M1: Metástase a distância } \\
\hline
\end{tabular}

Fonte: Cangussu IV, et al., 2020. Dados extraídos de Pape UF, et al., 2016. 
TNE de apêndice menores que $1 \mathrm{~cm}$ de diâmetro, segundo a classificação supracitada (ENETES), apresentam maiores taxas de sobrevida e boa resposta após realização de apendicectomia (PAPE UF, et al., 2016). Para pacientes com TNE do apêndice bem diferenciado, entre 1 e $2 \mathrm{~cm}$ e margens livres, não há consenso sobre o seguimento e a necessidade ou não de reintervenção cirúrgica (PAPE UF, et al., 2016; GROZINSKY-GLASBERG S, et al., 2013), sendo sugerido a realização de tomografia computadorizada ou ressonância magnética para avaliar comprometimento linfonodal ou doença a distância, a fim de definir conduta (PAPE UF, et al., 2016).

Por outro lado, pacientes com TNE em base do apêndice, tumores maiores que $2 \mathrm{~cm}$, invasão profunda do mesoapêndice ou angiolinfática ou margens comprometidas, apresentam maior risco de recorrência da doença e, por isso, devem ser investigados e enquadrados como candidatos a nova abordagem cirúrgica através da realização de uma hemicolectomia direita (PAPE UF, et al., 2016; CORREA NETO IJF, et al., 2014; GROZINSKY-GLASBERG S, et al., 2013). Em casos de tumores avançados com invasão peritoneal e/ou de órgão adjacentes, acometimento linfonodal e presença de metástase a distância é indicado tratamento multidisciplinar. No presente relato de caso, o tamanho do tumor foi a indicação para submissão da paciente a hemicolectomia direita complementar (PAPE UF, et al., 2016; TANG LH, et al., 2008).

De acordo com a ENETS, não é mandatório o acompanhamento de pacientes com tumores menores que $1 \mathrm{~cm}$ que foram submetidos a apendicectomia com margens cirúrgicas livres (PAPE UF, et al., 2016; PAPE UF, et al., 2012). Pacientes portadores de tumores T1 (ENETS) ou T1a (AJCC) na ausência de fatores de riscos, que realizaram excisão do apêndice podem ser considerados curados (NCCN, 2016). Em pacientes com tumores maiores que $1 \mathrm{~cm}$, submetidos a hemicolectomia direita, sem fatores de risco, sem invasão e sem doença residual, o acompanhamento não é considerado mandatório.

Entretanto o acompanhamento regular é indicado em tumores de $1 \mathrm{a} 2 \mathrm{~cm}$ que apresentam risco de disseminação para linfonodos: tumor na base do apêndice, infiltração vascular, acometimento do mesoapêndice maior que $3 \mathrm{~mm}$ e grau intermediário de diferenciação (PAPE UF et al., 2016). O seguimento a longo prazo deve ser preconizado em situações com acometimento linfonodal, tumor em estágio avançado e invasão locorregional (PAPE UF, et al., 2016; TANG LH, et al., 2008). A hemicolectomia direita é considerada um procedimento seguro por alguns estudos, com baixas taxas de complicações. Em uma metanálise, foram encontradas taxas de complicações em torno de 11, 4\% para esse procedimento (DASKALAKIS K, et al., 2020).

Em contrapartida, uma análise retrospectiva demonstrou diferenças nos resultados de curto prazo entre os grupos submetidos a apendicectomia e a hemicolectomia. Embora as taxas de readmissão e de reoperação fossem baixas e semelhantes entre os grupos, as ressecções do cólon foram associadas a tempos operatórios mais longos e foram constatados aumento da perda de sangue e maior permanência no hospital em pacientes submetidos a hemicolectomia direita.

Além disso, a taxa de complicações perioperatórias, no grupo de ressecção do cólon, foi em torno de 5 vezes maior, o que atingiu significância estatística. Dessa forma, foi sugerido que uma abordagem cirúrgica mais conservadora pode fornecer resultados de sobrevida semelhantes, ao mesmo tempo que oferece complicações mais favoráveis e menor perfil de morbidade (CROWN A, et al., 2020). No caso em questão a paciente desenvolveu quadro de constipação intestinal no pós-operatório, porém apresentou boa evolução e boa resposta ao tratamento clínico.

Quanto a mortalidade em paciente submetidos a hemicolectomia direita, estudos indicam taxas reduzidas, por volta de $0,8 \%$ para quadros com metástase e $4,4 \%$ para casos sem metástase. Nesta mesma análise foi possível observar que a ocorrência de metástases em pacientes submetidos a cirurgia curativa, sendo apendicectomia ou hemicolectomia, parece não influenciar as taxas de sobrevida (DASKALAKIS K, et al., 2020). Entretanto, a presença de tumores maiores que $2 \mathrm{~cm}$ é considerada indicação de ressecções cirúrgicas mais extensas, através da hemicolectomia direita (MOERTEL CG, et al., 1987). O seguimento peri e pósoperatório dos pacientes apresenta alguns desafios como o déficit de biomarcadores validados para seleção de candidatos a hemicolectomia e monitoramento (DASKALAKIS K, et al., 2020). 
Os TNE do apêndice são neoplasias de difícil diagnóstico pré-operatório, que devem ser bem avaliadas por estudo anatomopatológico e imuno-histoquímico devido a necessidade de complementação de tratamento em alguns casos. A biópsia de congelação é fundamental em alguns casos podendo evitar uma segunda intervenção, como no caso descrito. A hemicolectomia direita está bem indicada nos casos com fatores de risco e deve ser avaliada e discutida com o paciente nos casos sem consenso na literatura, dessa forma, justificam-se mais pesquisas na área com intuito de contemplar estes pacientes e, dessa forma, produzir a melhor conduta para estes casos fora do consenso.

\section{REFERÊNCIAS}

1. ALEXANDRAKI KI, et al. Appendiceal neuroendocrine neoplasms: diagnosis and management. Endocrine-related cancer, 2016; 23(1): R27-41.

2. AMR B, et al. Management and outcomes of appendicular neuroendocrine tumours: Retrospective review with 5-year followup. European Journal of Surgical Oncology, 2015; 41(9): 1243-1246.

3. BAYHAN Z, et al. Appendix Neuroendocrine Tumor: Retrospective Analysis of 4026 Appendectomy Patients in a Single Center. Emergency medicine international, 2020: 4030527.

4. BOUDREAUX JP, et al. North American Neuroendocrine Tumor Society (NANETS). The NANETS consensus guideline for the diagnosis and management of neuroendocrine tumors: well-differentiated neuroendocrine tumors of the Jejunum, lleum, Appendix, and Cecum. Pancreas, 2010; 39(6): 753-766.

5. CONNOR SJ, et al. Appendiceal tumors: retrospective clinicopathologic analysis of appendiceal tumors from 7,970 appendectomies. Diseases of the Colon \& Rectum, 1998; 41(1): 75-80.

6. CORREA NETO IJF, et al. Carcinoid tumor of cecal appendix: one-year incidence at the Santa Marcelina Hospital. Journal of Coloproctology, 2014; 34(4): 245-249.

7. CROWN A, et al. Appendiceal Neuroendocrine Tumors: Does Colon Resection Improve Outcomes? Journal of gastrointestinal surgery: official journal of the Society for Surgery of the Alimentary Tract., 2020; 24(9): 2121-2126.

8. DASARI A, et al. Trends in the Incidence, Prevalence, and Survival Outcomes in Patients With Neuroendocrine Tumors in the United States. JAMA oncology, 2017; 3(10): 1335-1342.

9. DASKALAKIS K, et al. The risk of lymph node metastases and their impact on survival in patients with appendiceal neuroendocrine neoplasms: a systematic review and meta-analysis of adult and paediatric patients. Endocrine, 2020; 67(1):
20-34.

10. DESCHAMPS D, COUVELARD A. Endocrine Tumors of the Appendix: A Pathologic Review. Archives of pathology \& laboratory medicine, 2010; 134(6): 871-875.

11. FERNANDES LC, et al. Diagnóstico e tratamento de tumores carcinoides do trato digestivo. Revista da Associação Médica Brasileira, 2002; 48(1): 87-92.

12. GAETKE-UDAGER K, et al. Beyond acute appendicitis: imaging and pathologic spectrum of appendiceal pathology. Emergency radiology, 2014; 21(5): 535-42.

13. GROZINSKY-GLASBERG S, et al. Current size criteria for the management of neuroendocrine tumors of the appendix: are they valid? Clinical experience and review of the literature. Neuroendocrinology, 2013; 98(1): 31-37.

14. HATCH QM, GILBERT EW. Appendiceal Neoplasms. Clinics in colon and rectal surgery, 2018; 31(5): $278-287$.

15. LOPES JÚNIOR AG, et al. Tumor do apêndice vermiforme. Revista do Colégio Brasileiro de Cirurgiões, 2001; 28(3): 228229.

16. MODLIN IM, SANDOR A. An analysis of 8305 cases of carcinoid tumors. Cancer, 1997; 79(4): 813-29.

17. MOERTEL CG, et al. Carcinoid tumor of the appendix: treatment and prognosis. The New England journal of medicine, 1987; 317 (27): 1699-1701.

18. MOERTEL CL, et al. Carcinoid tumor of the appendix in the first two decades of life. Journal of pediatric surgery, 1990; 25(10): 1073-1075.

19. MORIS D, et al. Neuroendocrine Neoplasms of the Appendix: A Review of the Literature. Anticancer research, 2018; 38(2): 601-611.

20. NCCN - National Comprehensive Cancer Network. Clinical practice guidelines in oncology: neuroendocrine tumors (version 2.2016). Disponível em: https://www.nccn.org/. Acesso em: 20 set. 2020.

21. NUSSBAUM DP, et al. Management of 1 - to 2 -cm carcinoid tumors of the appendix: Using the National Cancer Data Base to address controversies in general surgery. Journal of the American College of Surgeons, 2015; 220(5): 894-903.

22. PAPE UF, et al. ENETS Consensus Guidelines for Neuroendocrine Neoplasms of the Appendix (Excluding Goblet Cell Carcinomas). Neuroendocrinology, 2016; 103(2): 144-52.

23. PAPE UF, et al. ENETS Consensus Guidelines for the management of patients with neuroendocrine neoplasms from the jejuno-ileum and the appendix including goblet cell carcinomas. Neuroendocrinology, 2012; 95 (2): 135-56.

24. SAHAN EK, et al. P53, KI-67, CD117 expression in gastrointestinal and pancreatic neuroendocrine tumours and evaluation of their correlation witth clinicopathological and prognostic parameters. The Turkish journal of gastroenterology: the official journal of Turkish Society of Gastroenterology, 2015; 26(2):104-111.

25. SANDOR A, MODLIN IM. A retrospective analysis of 1570 appendiceal carcinoids. The American journal of gastroenterology, 1998; 93(3): 422-428.

26. SHAW PA. The topographical and age distributions of neuroendocrine cells in the normal human appendix. The Journal of pathology, 1991; 164(03): 235-239.

27. SILVA RL, et al. Tumor neuroendócrino do apêndice cecal: experiência do Instituto Nacional de Câncer. Revista Brasileira de Cancerologia, 2010; 56(4): 463-470.

28. SPANOS CP, KAISER AM. Appendiceal Neoplasms. The ASCRS Textbook of Colon and Rectal Surgery. Springer, 2016 : 617-629.

29. TANG LH, et al. Pathologic classification and clinical behavior of the spectrum of goblet cell carcinoid tumors of the appendix. The American journal of surgical pathology, 2008; 32(10): 1429-1443.

30. TOMIOKA K, et al. Primary neuroendocrine carcinoma of the appendix: a case report and review of the literature. Anticancer research, 2013; 33(6): 2635-2638.

31. WILLIAM Z, et al. Surgical management of appendicular carcinoids. World Journal of Pathology, 2013; 2: 7-13.

32. YAO JC, et al. One hundred years after "carcinoid": epidemiology of and prognostic factors for neuroendocrine tumors in 35,825 cases in the United States. Journal of clinical oncology: official journal of the American Society of Clinical Oncology, 2008; 26(18): 3063-3072. 